made in individuals presenting with dysphagia and/or food bolus impaction, based on typical endoscopic findings (expressed as EREFS score determining the severity of 5 endoscopic findings: oedema, rings, exudates, furrows and strictures) and confirmative histology (>15 eosinophils per highpower field). Treatments include unlicensed swallowed fluticasone via a metered-dose inhaler, proton-pump inhibitors (PPI) and elimination diets, though clinico-histological remission is variable. Orodispersible budesonide (Jorveza) is the first licensed therapy for EoE and has recently been approved by NICE for induction treatment in the UK. This is an audit of the first six months' experience of its use in our centre.

Methods Pharmacy prescription records were used to identify patients who had been prescribed orodispersible budesonide between September 2020 and March 2021. Case note review was performed to document treatment history, baseline endoscopic and histological findings, clinical and endoscopic response, follow-up and adverse effects.

Results 27 patients were identified; 78\% were male, with a mean age of 45 years (range 23-74). 85\% were symptomatic with dysphagia and $44 \%$ had a history of food bolus impaction. $93 \%$ of patients had failed medical therapy before starting orodispersible budesonide and $63 \%$ had failed an elimination diet. All patients had a baseline endoscopy prior to starting treatment, with a mode EREFS score of 3 . The majority of patients $(78 \%)$ were treated with a 6 week course and the remainder received 12 weeks, with 6 patients $(22 \%)$ going on to a maintenance dose.

$94 \%$ of patients had a clinical review within 12 weeks of the original prescription. Overall, $86 \%$ achieved symptomatic remission. $56 \%$ of patients had a follow-up endoscopy after at least 6 weeks of treatment, with some impact from reduction of endoscopy services during the second wave of the COVID19 pandemic. Of those who had a follow-up endoscopy, 50\% achieved endoscopic remission, as defined by an EREFS of 0 . $61 \%$ achieved histological remission, as defined by an eosinophil count of $<15$ per high-power field (hpf). The mean drop in peak eosinophil count was 58 per hpf (range 4-117).

Three patients reported adverse effects with two reporting new onset gastro-oesophageal reflux symptoms and one developing acne.

Conclusions Our tertiary single-centre experience demonstrates good adherence with NICE guidance regarding use of orodispersible budesonide in EoE. Clinical, endoscopic and histological remission is achieved in the majority of cases, in line with published evidence supporting its use.

\section{PTH-77 BIOPSIES FOR GRADE D OESOPHAGITIS; DO WE NEED THEM?}

Tamara Glaister*, Julia F Simons, Suneil A Raju, John M Hebden. Academic Unit of Gastroenterology, Royal Hallamshire Hospital, Sheffield, UK

\subsection{6/gutjnl-2021-BSG.256}

Introduction British Society of Gastroenterology guidelines state all patients with Los Angeles (LA) grade D oesophagitis should be biopsied and re-evaluated at 6 weeks to exclude dysplasia and malignancy. There is a paucity of data showing outcomes. We present the largest data on the clinical use of biopsies in patients with grade D oesophagitis.

Methods All patients with LA grade D oesophagitis were identified between January 2018 and December 2019 in a tertiary teaching hospital. Further data was collected from case notes and on subsequent endoscopies. Patient outcomes were followed up until May 2021.

Results In total, 132 patients were identified with grade D oesophagitis on index gastroscopy [median age 69 years, (IQR 55 - 78 years), 59\% male]. Indications for index gastroscopy were suspected gastrointestinal bleed (37\%), dysphagia (21\%), anaemia (15\%) and nausea (14\%).

Oesophageal biopsies were taken in $21 \%$ of index gastroscopies median 3 (IQR 2-5). Biopsies identified oesophageal ulceration (37\%), reflux oesophagitis (26\%), Barrett's (19\%) and erosive oesophagitis (15\%). No dysplasia or carcinoma was identified histologically or endoscopically.

Repeat gastroscopy was performed in $57 \%$ of patients at median 55 days (IQR 42-70 days) and 16\% reported on-going grade D oesophagitis. Biopsies were taken during repeat gastroscopies in $33 \%$ of cases. No dysplasia or carcinomas were identified, however $23 \%$ of patients were found to have Barrett's oesophagus which was not previously identified. During the follow-up period no oesophageal cancer was identified.

Conclusions No dysplasia or carcinoma was discovered at index or follow-up gastroscopy, however, repeat gastroscopies are still required to exclude other diagnoses. Adherence to biopsy guidelines is low, as is follow-up gastroscopy for patients with LA grade D oesophagitis though during the follow-up period, no oesophageal cancers were identified.

\section{PTH-78 COMPUTATIONAL COLOUR CONTRAST-ENHANCEMENT IMPROVES ENDOSCOPIC VISIBILITY OF OESOPHAGEAL SQUAMOUS DYSPLASIA AND DETECTION IN AI-BASED SYSTEM}

${ }^{1}$ Xiaohong Gao, ${ }^{2}$ Stephen Taylor, ${ }^{3}$ Prof Wei Pang, ${ }^{4}$ Rui Hui, ${ }^{5}$ Prof Xin Lu, ${ }^{6}$ Investigators TGU Oxford, ${ }^{6}$ Barbara Braden*. 'Middlesex University, London, UK; ${ }^{2}$ University of Oxford, Oxford, UK; ${ }^{3}$ University of Heriot-Watt, Edinburgh, UK; ${ }^{4}$ Beijing Navy General Hospital, Bejing, China; ${ }^{5}$ Ludwig Institute, University of Oxford, Oxford, UK; ${ }^{6}$ Translational Gastroenterology Unit, Oxford, Oxford, UK

\subsection{6/gutjnl-2021-BSG.257}

Introduction White light (WLI) endoscopy often misses precancerous oesophageal changes in squamous epithelium due to their only subtle visual differences to the surrounding normal mucosa. This paper aims to exploit computational enhancement of colour contrast from human perception point of view to increase the visibility of squamous dysplasia and to optimise a deep learning-based decision support system.

Methods 905 WLI images from 277 patients including histologically confirmed low grade (LGD), high grade dysplasia (HGD) or intramucosal squamous cancer (SCC) together with their corresponding narrow band (NBI) were evaluated. By applying the Commission Internationale de L'Eclairage (CIE) colour appearance model CIECAM02 to represent an image using human perceptual attribute correlates, each pixel of an image was enhanced and converted back to RGB values to display contrasted images. Colour differences between dysplastic regions and normal surrounding mucosa were measured in the original images and in the contrast-enhanced images. These contrast-enhanced images were also embedded to train a deep learning-based detection system that was tested in an external independent cohort of 112 patients (70 normal).

Results Through the modelling of colour appearance and contrast enhancement based on human colour vision, a real-time endoscopy decision support system was developed that works 
Abstract PTH-78 Table 1

\begin{tabular}{|c|c|c|c|c|c|c|c|c|}
\hline & $\Delta \mathrm{L}$ & $\Delta a^{*}$ & $\Delta b^{*}$ & $\Delta \mathrm{E}_{\mathrm{L}^{*} \mathrm{a}^{*} \mathrm{~b}^{*}}$ & $\Delta \mathrm{L}_{\text {CAM }}$ & $\Delta C_{\text {CAM }}$ & $\Delta \mathrm{H}_{\text {CAM }}$ & $\Delta \mathrm{E}_{\text {CAM }}(\%)$ \\
\hline WLI & 5.76 & 6.23 & 7.38 & 11.60 & 4.83 & 9.23 & 19.30 & 13.12 \\
\hline Enhanced & 7.74 & 8.48 & 8.44 & 14.46 & 6.62 & 12.29 & 27.48 & 18.35 \\
\hline \multicolumn{9}{|l|}{ WLI } \\
\hline NBI & 8.17 & 10.59 & 9.50 & 17.52 & 9.57 & 3.73 & 6.25 & 10.82 \\
\hline Enhanced & 10.23 & 16.22 & 21.56 & 32.53 & 12.00 & 10.06 & 95.32 & 33.60 \\
\hline NBI & & & & & & & & \\
\hline
\end{tabular}

in WLI and NBI (multi-modal) and classifies into LGD, HGD and SCC (multi-class).

The averaged colour difference (measured by CIECLAB $\square$ E) between dysplastic regions and surroundings for the post-processed contrasted images increased from 11.60 to 14.46 for WLI and from 17.52 to 32.53 for NBI. With the addition of contrast-enhanced WLI images to the training of the deep learning system, its sensitivity, specificity and accuracy for detecting LGD increased from $75 \%, 88.2 \%$ and $81.4 \%$ to $82.7 \%, 96.9 \%$ and $90.6 \%$ respectively and were $88.3 \%$, $94.4 \%$ and $92.6 \%$ for the classification of SCC, HGD and LGD.

Table The colour differences computed using CIEL*a*b* between each LGD and its surrounding mucosa for original WLI and NBI and their enhanced counterparts for all LGD samples.

Conclusion Computational contrast-enhancement facilitates early identification of oesophageal neoplasia during visual inspection at endoscopy and significantly improves the performance of a deep learning system.

\section{Gastroduodenal}

\section{PTH-79 POST-ERADICATION RETESTING FOR HELICOBACTER PYLORI INFECTION IN PATIENTS UNDERGOING UPPER GASTRO-INTESTINAL ENDOSCOPY: COMPLIANCE WITH GUIDELINES}

${ }^{1}$ Mohammad Alradhawi*, ${ }^{1}$ Nekisa Zakeri, ${ }^{2}$ Rupert Negus, ${ }^{2}$ Damien Mack, ${ }^{1}$ Marsha Y Morgan. 'University College London, London, UK; ${ }^{2}$ Royal Free Hospital, London, UK

\subsection{6/gutjpl-2021-BSG.258}

Introduction Failure to eradicate Helicobacter pylori $(H p)$ infection increases the risk of subsequent peptic ulcer disease (PUD) and gastric cancer (GC). Therefore, confirmation of successful eradication is vital, although guidelines differ in their recommendations (Table 1). The prevailing opinion is that retesting should take place 4-8 weeks after treatment completion using the ${ }^{13} \mathrm{C}$-urea breath test (UBT). This study aimed to evaluate compliance with post-eradication testing guidelines in $H p$ positive patients.

Methods Compliance with post-eradication $H p$ testing within the recommended 4-8 week timeframe of treatment completion was retrospectively assessed in 408 patients (60\% female; mean $\pm 1 S D$ age $40.9 \pm 20.8$ yr) with recurrent/previous $H p$ infection who underwent upper GI endoscopy at the Royal Free Hospital (RFH) London between January 2009 and December 2019. Data were collected from GP referral letters, the patients' electronic records, pathology and endoscopy results.

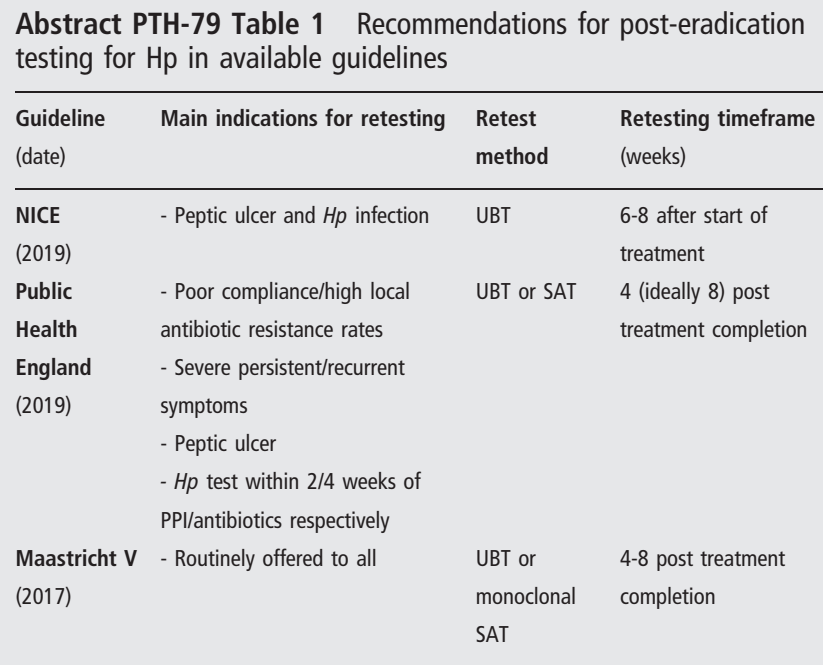

Results Of the 408 patients, 118 (28.9\%) were $H p$ positive on culture; 91/118 (77.1\%) received eradication treatment. Posteradication data were available for 90 patients; of whom 65 (72.2\%) underwent $H p$ testing with UBT (38: 58.5\%), stool antigen test (SAT) (23: 35.4\%), CLO test in one $(1.5 \%)$ or unrecorded in three. Of the 65 tested, results were available for 58; persistent infection was identified in 37 (63.8\%). Only $27.3 \%$ were retested within the recommended 4-8 week time frame; the median time for testing was 16 weeks.

Conclusion Compliance with post-eradication $\mathrm{Hp}$ testing was very poor. Only $77.1 \%$ of eligible patients received eradication treatment; of these only $72.2 \%$ underwent post-eradication testing and only a quarter within the recommended timeframe. Persistent infection was identified in $63.8 \%$ of those tested; current practice is therefore failing patients and necessitates the urgent adoption of a planned 'test, treat, and confirm eradication' care package.

*joint first authors

UBT ${ }^{13} \mathrm{C}$-urea breath test; SAT: stool antigen testing; PPI: Proton pump inhibitor

\section{PTH-80 COMPARISON BETWEEN GLASGOW BLATCHFORD, ROCKALL, AND AIMS65 SCORING SYSTEMS IN PREDICTING OUTCOME OF GASTROINTESTINAL BLEEDING}

Elaf Abdalla*, Elaf Abdalla, Omer Kheir, Hala Ibrahim, Salma Barakat. Ncgld, Khartoum, Sudan

10.1136/gutjnl-2021-BSG.259 